International Journal of Pure and Applied Mathematics

Volume 110 No. 1 2016, 71-82

ISSN: 1311-8080 (printed version); ISSN: 1314-3395 (on-line version)

url: http://www.ijpam.eu

doi: 10.12732/ijpam.v110i1.8

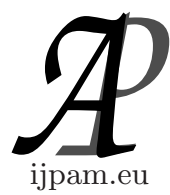

\title{
A NOTE ON P-SYMMETRIC OPERATORS
}

\author{
Said Bouali ${ }^{1}$, Mohamed Ech-Had ${ }^{1}$, Adil Zouaki ${ }^{1}$, Youssef Bouhafsi ${ }^{2} \S$ \\ 1,2,3 Laboratory of Analysis, Geometry and Applications (LAGA) \\ Department of Mathematics \\ Faculty of Science \\ Ibn Tofail University, B.P. 133, Kénitra, MOROCCO \\ ${ }^{2}$ Laboratory of Fundamental Mathematics (LMF) \\ Department of Mathematics \\ Faculty of Science \\ Chouaïb Doukkali University \\ B.P. 20, El Jadida, MOROCCO
}

\begin{abstract}
Let $L(H)$ denote the algebra of operators on a complex infinite dimensional Hilbert space $H$ into itself. In this paper, we study the class of operators $A \in L(H)$ which satisfy the following property, $A T=T A$ implies $A T^{*}=T^{*} A$ for all $T \in C_{1}(H)$ (trace class operators). Such operators are called p-symmetric. We establish some basic properties on the class of p-symmetric operators. We obtain new results concerning the intersection of the closure of $R\left(\delta_{A}\right)$, the range of the derivation $\delta_{A}(X)=A X-X A$, and the commutant $\{A\}^{\prime}$ of $A$. We introduce the class of essentially d-symmetric operators. Some open problems are also presented.
\end{abstract}

AMS Subject Classification: 47A30, 47B47, 47B20, 47B10

Key Words: Derivation, d-symmetric operator, p-symmetric operator, subnormal operator.

\section{Introduction}

Let $H$ be a complex infinite dimensional Hilbert space, and let $L(H)$ denote the

Received:

Revised:

September 4, 2016

Published:

October 21, 2016 (c) 2016 Academic Publications, Ltd. url: www.acadpubl.eu

${ }^{\S}$ Correspondence author 
algebra of all bounded linear operators acting on $H$ into itself. Given $A \in L(H)$, the derivation induced by $A$ is the bounded operator $\delta_{A}$ defined on $L(H)$ by $\delta_{A}(X)=A X-X A$.

Let $R\left(\delta_{A}\right)$ denote the range of $\delta_{A}$ and $\overline{R\left(\delta_{A}\right)}$ its uniform closure. An operator $A$ in $L(H)$ is called d-symmetric if $\overline{R\left(\delta_{A}\right)}=\overline{R\left(\delta_{A^{*}}\right)}$. The concept of d-symmetric was introduced by Bunce and Williams.

In [2] J. Anderson, J.W. Bunce, J.A. Deddens and J.P. Williams show that, if $A$ is d-symmetric then $A T=T A$ implies $A^{*} T=T A^{*}$ for every $T \in C_{1}(H)$ (trace class operators). Examples of d-symmetric operators include the normal operators and isometries. The properties of such operators have been much studied in [2], [10], [14], [15], [17].

In this paper, we study the class of operators $A \in L(H)$ which satisfy the following property, $A T=T A$ implies $A^{*} T=T A^{*}$ for all $T \in C_{1}(H)$. Such operators are termed p-symmetric. The class of p-symmetric operators has been considered in a number of papers, see for examples [4], [5], [6], [7], [8], [13].

It is shown that if $P(A)$ is cyclic subnormal for some polynomial $P$, then $A$ is d-symmetric. In this case, we also prove that the range $R\left(\delta_{A}\right)$ is orthogonal to the kernel $\operatorname{ker}\left(\delta_{A}\right)$ of $\delta_{A}$ for the usual operator norm. We establish some basic properties on the class of p-symmetric operators. We obtain new results concerning the intersection of the closure of $R\left(\delta_{A}\right)$ and the commutant $\{A\}^{\prime}$ of $A$.

Finally, we introduce the class of essentially d-symmetric operators. We close the paper with some open problems.

In addition to the notation already introduced, we shall use the following further notation. Given $X \in L(H)$, we shall denote the kernel, the orthogonal complement of the kernel, the closure of the range and the restriction to an invariant subspace $M$ of $X$ by $\operatorname{ker}(X), \operatorname{ker}^{\perp}(X), \overline{R(X)}$ and $X \mid M$ respectively. ${\overline{R\left(\delta_{X}\right)}}^{w}$ will denote the weak closure and $\overline{R\left(\delta_{X}\right)} w^{*}$ will denote the ultra- weak closure of $R\left(\delta_{X}\right)$ the range of $\delta_{X}$. The spectrum, the essential spectrum and the point spectrum of $X$ will be denoted by $\sigma(X), \sigma_{e}(X)$ and $\sigma_{p}(X)$. Let $K(H)$ be the algebra of all compact operators on $H$, and let $\pi(A)$ be the image of $A \in L(H)$ under the canonical mapping $\pi: L(H) \longrightarrow \mathcal{C}(H)=L(H) \mid K(H)$.

\section{Preliminaries}

Definition 1. An operator $A \in L(H)$ is called d-symmetric, if $\overline{R\left(\delta_{A}\right)}=$ $\overline{R\left(\delta_{A^{*}}\right)}$. 
Theorem 2. ([2]) If $A \in L(H)$, then the following two statements are equivalent:

(1) $A$ is d-symmetric.

(2) (i) $[A]$, the corresponding element of the Calkin algebra, is d-symmetric, and (ii) $A T=T A$ and $T \in C_{1}(H)$ implies $A^{*} T=T A^{*}$.

Definition 3. Let $A \in L(H)$. $A$ is called p-symmetric if: $A T=T A$ and $T \in C_{1}(H)$ implies $A^{*} T=T A^{*}$.

Theorem 4. ([4]) Let $A \in L(H)$, then:

(1) $A$ is p-symmetric if and only if ${\overline{R\left(\delta_{A}\right)}}^{w^{*}}$ is self-adjoint.

$(2) \mathcal{S}(H)$ (the set of p-symmetric operators) is self-adjoint.

Proposition 2.1. ([4]) Let $A \in L(H)$. If there exist nonzero vectors $f, g \in H$ such that:

(1) $A f=\lambda f$ and,$A^{*} f \neq \bar{\lambda} f$.

(2) $A^{*} g=\bar{\lambda} g$.

Then, $A$ is not p-symmetric.

Example 2.1. Let $\left(e_{n}\right)_{n}$ be an orthonormal basis for $H$. Let $H_{\circ}=$ vect $\left\{e_{1}, e_{2}, e_{3}\right\}$ and set

$$
A_{\circ}=\left(\begin{array}{ccc}
i & 1 & 0 \\
0 & -i & 0 \\
0 & 1 & 1
\end{array}\right) \in L\left(H_{\circ}\right)
$$

We define the operator $A=A_{\circ} \oplus I$ with respect to decomposition $H=H_{\circ} \oplus H_{\circ}^{\perp}$. It is easily seen that, $A e_{1}=i e_{1}, A^{*} e_{1}=-i e_{1}+e_{2} \neq-i e_{1}$, and $A^{*}\left(2 e_{1}+i e_{2}\right)=$ $(-i)\left(2 e_{1}+i e_{2}\right)$. Hence, it follows that $A$ is not p-symmetric.

Example 2.2. Let $H=H_{\circ} \oplus H_{1}$, where $H_{\circ}$, and $H_{1}$ are complex separable Hilbert space. Define $T=A \oplus S$, where $A$ is p-symmetric and strict contraction $\|A\|<1$, and $S$ is the unilateral or bilateral shift: $S e_{n}=e_{n+1}, n \in \mathbb{N}(\mathbb{Z})$ where $\left(e_{n}\right)_{n}$ is an orthonormal basis for $H_{1}$. It is clearly seen that $A$ and $S$ are p-symmetric and $\sigma(A) \cap \sigma(S)=\phi$. Then $T$ is a p-symmetric operator. 


\section{Main Results}

Definition 5. A vector $e_{\circ} \in H$ is cyclic for $A \in L(H)$, if $H$ is the smallest invariant subspace for $A$ that contains $e_{0}$. The operator $A$ is said to be cyclic if it has a cyclic vector.

Definition 6. An operator $A \in L(H)$ is subnormal, if there exists a Hilbert space $H_{1}$ and a normal operator $N \in L\left(H_{1}\right)$, such that $H$ is a subspace of $H_{1}$ and $A f=N f$ for all $f \in H$.

Theorem 7. Let $A \in L(H)$, and suppose that $P(A)$ is cyclic subnormal for some polynomial $P$. Then, $A$ is a $d$-symmetric operator.

Proof. Let $T \in C_{1}(H)$ such that $A T=T A$, then $P(A) T=T P(A)$. Since $P(A)$ is a cyclic subnormal operator, it follows from Yoshino's result [22] that $T$ is subnormal and therefore normal. The Putnam-Fuglede theorem gives $A^{*} T=T A^{*}$. Consequently, $A$ is p-symmetric.

The condition $P(A) A=A P(A)$ implies that $A$ is subnormal, hence $A$ is essentially hyponormal. We have $\pi[P(A)]=P[\pi(A)]$, then $P[\pi(A)]$ is normal in $\mathcal{C}(H)$.

There exists a Hilbert space $H_{\circ}$ and a $*$-isometric isomorphism $\psi: \mathcal{C}(H) \longrightarrow$ $L\left(H_{\circ}\right)$ preserving order. Therefore $P[\psi \circ \pi(A)]=\psi[P(\pi(A))]$ and $P(\pi(A))$ is normal. Hence, $\psi[P(\pi(A))]$ is polynomially normal and hyponormal in $L\left(H_{\circ}\right)$. Thus $\psi[\pi(A)]$ is normal [16], and so $\pi(A)$ is normal. It follows that $A$ is essentially d-symmetric. Then $A$ is d-symmetric operator, as desired.

Remark 3.1. In $[2]$ it is proved that an operator $A \in L(H)$ is d-symmetric if and only if

$$
A^{*} A-A A^{*} \in \mathcal{C}(A)=\left\{C \in L(H): C L(H)+L(H) C \subseteq \overline{R\left(\delta_{A}\right)}\right\} .
$$

Let $U$ be an isometry. Then it follows from Theorem 2 [19] that

$$
\left(I-U U^{*}\right) L(H) \subset R\left(\delta_{U}\right) .
$$

On the other hand if $Y \in L(H)$, note that the operator $X=\Sigma_{k=0}^{\infty} U^{k} P Y P U^{*(k+1)}$ is bounded and that

$$
\delta_{U}\left(U^{*} Y P-X\right)=Y P .
$$

Hence

$$
L(H)\left(I-U U^{*}\right) \subset R\left(\delta_{U}\right)
$$

Thus we get

$$
\left(I-U U^{*}\right) L(H)+L(H)\left(I-U U^{*}\right) \subset R\left(\delta_{U}\right) .
$$


Theorem 2.1[2], implies that $U$ is d-symmetric.

Example 3.1. Let $H_{\circ}=H \oplus H$, and define the operator $A=\left(\begin{array}{cc}0 & I \\ 0 & 0\end{array}\right)$. If we consider $T=\left(\begin{array}{cc}C & D \\ 0 & C\end{array}\right) \in C_{1}\left(H_{\circ}\right)$ such that $D \neq 0$. A simple calculation shows that $A^{2}=0$ and $A T=T A$. On the other hand $A^{*} T-T A^{*}=$ $\left(\begin{array}{cc}-D & 0 \\ 0 & D\end{array}\right) \neq 0$. and so $A$ is not p-symmetric.

We are now in a position to extend this results. More precisely:

Proposition 3.1. Let $A \in L(H)$. If $A$ is a nilpotent operator, then $A$ is not p-symmetric.

Proof. Let $n>1$ be the smallest integer such that $A^{n}=0$ and $A^{n-1} \neq 0$. Let $e \in H$ such that $f=A^{n-1} e \neq 0$. Then, a simple calculation shows that $A f=0$ and $A^{*} f \neq 0$. We can also choose $k \in H$ be such that $g=A^{* n-1} k \neq 0$. Note that $w=A^{*} f \neq 0$ where $w \perp f$. Define an operator $X \in L(H)$, as follows $X=\|w\|^{-2}(g \otimes w)$. Then for any $Y \in L(H)$

$$
<\left(A^{*} X-X A^{*}\right) f, g>=<X w, g>=-\|g\|^{2},
$$

and $<(A Y-Y A) f, g>=0$.

Moreover, suppose that $A^{*} X-X A^{*} \in \overline{R\left(\delta_{A}\right)} w^{*}$, then there exists a generalized sequence $\left(Y_{\alpha}\right)_{\alpha}$ in $L(H)$ such that

$$
<\left(A Y_{\alpha}-Y_{\alpha} A\right) x, y>\longrightarrow<\left(A^{*} X-X A^{*}\right) x, y>
$$

for all $x, y \in H$. Which implies

$$
0=<\left(A Y_{\alpha}-Y_{\alpha} A\right) f, g>\longrightarrow<\left(A^{*} X-X A^{*}\right) f, g>=-\|g\|^{2} .
$$

Then, it follows that $A^{*} X-X A^{*} \notin \overline{R\left(\delta_{A}\right)} w^{*}$, thus $\overline{R\left(\delta_{A}\right)} w^{*}$ is not self-adjoint. This proves that $A$ is not p-symmetric.

An interesting consequence of the above theorem is the following:

Corollary 8. Let $A \in L(H)$. If $P(A)$ is normal, isometric, co-isometric or cyclic subnormal for some polynomial $P$, then $\overline{R\left(\delta_{A}\right)} \cap\{A\}^{\prime}$ contains nonzero p-symmetric operator. 
Proof. Suppose $A X_{n}-X_{n} A \longrightarrow T$ and that $T$ is a p-symmetric operator in $\{A\}^{\prime}$. If $P^{(k)}$ denotes the $k$-derivative of $P$, then

$$
P^{(k)}(A) X_{n}-X_{n} P^{(k)}(A) \longrightarrow P^{(k+1)}(A) T .
$$

In particular, $P^{(1)}(A) T \in \overline{R\left(\delta_{P(A)}\right)} \cap\{P(A)\}^{\prime}$, so that $P^{(1)}(A) T=0$, since $P(A)$ is normal, isometric, co-isometric or cyclic subnormal. Also,

$$
P^{(1)}(A) X_{n}-X_{n} P^{(1)}(A) \longrightarrow P^{(2)}(A) T .
$$

Hence, $P^{(2)}(A) T^{3}=0$. By repeating the same argument it follows that, $P^{(m)}(A) T^{m+1}=0$, where $m$ is the degree of $P$. Thus $T^{m+1}=0$ and so $T=0$, since it is p-symmetric.

Theorem 9. Let $A \in L(H)$, and suppose that $P(A)$ is cyclic subnormal for some polynomial $P$. Then,

$$
\left\|\delta_{A}(X)+T\right\| \geq\|T\|
$$

for all $X \in L(H)$ and for every operator $T$ satisfying $A T=T A$.

Proof. Let $T \in L(H)$ be such that $A T=T A$. So we have $A P(A)=P(A) A$ and $P(A) T=T P(A)$. Since $P(A)$ is a cyclic subnormal operator, then it follows from Yoshino's Result [22] that $T$ and $A$ are subnormal. Thus, it is sufficient to show that

$$
\left\|\delta_{A}(X)+T\right\| \geq|\lambda|
$$

for all $X \in L(H)$ and for every $\lambda \in \sigma(T)$.

The operator $T$ being subnormal, then it is well known that: $\sigma(T)=\sigma_{p}(T) \cup$ $\sigma_{e}(T)($ see $[9])$.

Let $\lambda \in \sigma(T)$, we consider the following cases for the location of $\lambda$ :

Case 1. (i) Suppose that $\lambda \in \sigma_{p}(T)$, such that $E=\operatorname{ker}(T-\lambda)$ is finite dimensional. The subspace $E$ is invariant under $A$ and $T$. Moreover, $A \mid E$ is normal, so $E$ reduces $A$. Let $H=E \oplus E^{\perp}$, then we can write $A=\left(\begin{array}{cc}B & 0 \\ 0 & C\end{array}\right)$ and $T=\left(\begin{array}{cc}\lambda & 0 \\ 0 & *\end{array}\right)$

For an operator $X=\left(\begin{array}{cc}Y & Z \\ R & S\end{array}\right)$, we have

$$
\left\|\delta_{A}(X)+T\right\|=\left\|\left(\begin{array}{cc}
\delta_{B}(Y)+\lambda & * \\
* & *
\end{array}\right)\right\| \geq\left\|\delta_{B}(Y)+\lambda\right\|
$$


$A$ is subnormal, then $A$ is a finite operator [21], it results that $B$ is also finite. Consequently, we obtain

$$
\left\|\delta_{A}(X)+T\right\| \geq|\lambda|
$$

for all $X \in L(H)$, and all $\lambda \in \sigma_{p}(T)$ such that $\operatorname{dim} \operatorname{ker}(T-\lambda)<\infty$.

(ii) If $\lambda \in \sigma_{p}(T)$ such that $\operatorname{dim} \operatorname{ker}(T-\lambda)=\infty$. Since $T$ is a subnormal operator then $\operatorname{dim} \operatorname{ker}(T-\lambda)^{*}=\infty$. It follows that $T-\lambda$ is not a Fredholm operator which is equivalent to $\lambda \in \sigma_{e}(T)$.

The remark [9 ,p.187] plays a vital role in the following case.

Case 2. If $\lambda \in \sigma_{e}(T)$. We shall divide this cases into two different steps.

(i) $T$ has no isolated eigenvalues of finite multiplicity.

The condition $A T=T A$ implies that $\pi(A) \pi(T)=\pi(T) \pi(A)$. Since $P(A)$ is a cyclic subnormal operator, then it results that $\pi(A)$ is normal. Hence $R\left(\delta_{\pi(A)}\right)$ is orthogonal to $\operatorname{ker}\left(\delta_{\pi(A)}\right)$. From this it follows that

$$
\left\|\delta_{A}(X)+T\right\| \geq\left\|\delta_{\pi(A)}(\pi(X))+\pi(T)\right\| \geq\|\pi(T)\|
$$

For all $X \in L(H)$. Since $T$ is subnormal and has no isolated eigenvalues of finite multiplicity, then we have $\|\pi(T)\|=r(\pi(T))$. Which implies that

$$
\left\|\delta_{A}(X)+T\right\| \geq|\lambda|
$$

For all $X \in L(H)$.

(ii) If $T$ has isolated eigenvalues of finite multiplicity. We consider the subspace $F=\bigvee\left\{\operatorname{ker}(T-\mu): \mu \in \sigma_{p}(T), \operatorname{dim} \operatorname{ker}(T-\mu)<\infty\right\}$. The condition $A T=T A$ implies that $T$ is subnormal. Since $T \mid F$ is a normal operator then $F$ reduces $T$. With respect to the decomposition $H=F \oplus F^{\perp}$, we have $T=$ $\left(\begin{array}{cc}T_{1} & 0 \\ 0 & T_{2}\end{array}\right)$.

It is easily seen that

$$
\left\|\delta_{A}(X)+T\right\| \geq\left\|\delta_{\pi(A)}(\pi(X))+\pi(T)\right\| \geq\|\pi(T)\| .
$$

On the other hand it is clear to check that $T$ is a Fredholm operator if and only if $T_{2}$ is a Fredholm operator. Consequently, We get $\sigma_{e}(T)=\sigma_{e}\left(T_{2}\right)$. By hypothesis we have $\lambda \in \sigma_{e}(T)=\sigma_{e}\left(T_{2}\right)$ and $T=T_{1} \oplus T_{2}$. Then it is obvious that $\|\pi(T)\| \geq\left\|\pi\left(T_{2}\right)\right\|$.

Since $T_{2}$ has no isolated eigenvalues of finite multiplicity, then it follows immediately that $\left\|\delta_{A}(X)+T\right\| \geq|\lambda|$ for all $X \in L(H)$. 
Indeed, we assert that

$$
\left\|\delta_{A}(X)+T\right\| \geq|\lambda|
$$

for all $X \in L(H)$ and all $\lambda \in \sigma_{e}(T)$.

Finally, we deduce that

$$
\left\|\delta_{A}(X)+T\right\| \geq\|T\|
$$

for all $X \in L(H)$ and all $T \in\{A\}^{\prime}$. This completes the proof.

The following result is now immediate from the preceding theorem.

Corollary 10. Let $A \in L(H)$. If $P(A)$ is cyclic subnormal for some polynomial $P$, then we have $\overline{R\left(\delta_{A}\right)} \cap\{A\}^{\prime}=\{0\}$ and $\overline{R\left(\delta_{A}\right)} \cap\left\{A^{*}\right\}^{\prime}=\{0\}$.

Remark 3.2. 1) If $A$ is a normal operator or isometric, then $\overline{R\left(\delta_{A}\right)} \cap$ $\left\{\{A\}^{\prime} \cup\left\{A^{*}\right\}^{\prime}\right\}=\{0\}([1])$.

2) The above result generalizes the Theorem 3 of Y. Ho [11].

Remark 3.3. Let $A \in L(H)$. It is well known from [5] that

$$
\overline{R\left(\delta_{A}\right)} \cap K(H)={\overline{R\left(\delta_{A}\right)}}^{w^{*}} \cap K(H) .
$$

Hence, if $A$ is normal, isometric, co-isometric or cyclic subnormal then every compact operator in $\overline{R\left(\delta_{A}\right)} w^{*} \cap\left\{\{A\}^{\prime} \cup\left\{A^{*}\right\}^{\prime}\right\}$ vanishes.

Corollary 11. Let $A \in L(H)$. in each of the following cases:

(1) If $A$ is normal operator.

(2) If $P(A)$ is cyclic subnormal operator for some polynomial $P$.

(3) If $A$ isometric or co-isometric.

(4)If $A$ is invertible such that $\left\|A^{-1}\right\|\|A\|=1$.

Then ${\overline{R\left(\delta_{A}\right)}}^{w} \cap\left\{\{A\}^{\prime} \cup\left\{A^{*}\right\}^{\prime}\right\}$ contains no nonzero compact operator.

Proof. (1) Suppose that $T \in{\overline{R\left(\delta_{A}\right)}}^{w} \cap\{A\}^{\prime}$ and that $T$ is a compact operator. $A$ is normal, by Putnam-Fuglede's theorem we have $A T^{*}=T^{*} A$, and hence $A|T|^{2}=|T|^{2} A$. Moreover, by the functional calculus $A|T|=|T| A$. Therefore, there exists an orthonormal basis $\left\{\phi_{n}\right\}=\left\{e_{k}\right\} \cup\left\{f_{m}\right\}$ of $H$, such that $\left\{f_{m}\right\}$ is an orthonormal basis of $\operatorname{ker}(|T|)$ and $\left\{e_{k}\right\}$ consists of common eigenvectors of $A$ and $|T|$. Since $T^{*} T \in \overline{R\left(\delta_{A}\right)} w^{w} \cap\{A\}^{\prime}$, then there exists a sequence $\left(X_{\alpha}\right)_{\alpha}$ of operators in $L(H)$ such that $A X_{\alpha}-X_{\alpha} A \longrightarrow T^{*} T$. It follows that

$$
<\left(A X_{\alpha}-X_{\alpha} A\right) \phi_{n}, \phi_{n}>\longrightarrow<T^{*} T \phi_{n}, \phi_{n}>
$$


for all $n$. Thus, we get $T e_{k}=0$ and $T f_{m}=0$ for all $k$ and $m$. Then, $T=0$, as desired.

(2) Let $T \in{\overline{R\left(\delta_{A}\right)}}^{w} \cap\{A\}^{\prime}$. Since $A$ commutes with $T$, it follows that $P(A) T=T P(A)$, and $P(A)$ is cyclic subnormal, then we get from Yoshino's result [22] that $T$ is subnormal and therefore normal [3]. Hence, $T^{*} T \in{\overline{R\left(\delta_{A}\right)}}^{w} \cap$ $\{A\}^{\prime}$, it is clear from [18, Theorem 3], that $T=0$.

We consider now cases (3) and (4), let $T \in{\overline{R\left(\delta_{A}\right)}}^{w} \cap\{A\}^{\prime}$. Since $A T=T A$ and $T \in K(H)$, it follows from [18,Theorem 2.2] that $A T^{*}=T^{*} A$. Then we get $T^{*} T \in{\overline{R\left(\delta_{A}\right)}}^{w} \cap\{A\}^{\prime}$. Applying [19, Theorem 3], we conclude that $\sigma\left(T^{*} T\right)=\{0\}$, hence $T=0$.

Definition 12. An operator $A \in L(H)$ is called essentially d-symmetric if $\pi(A)$, its corresponding element of the Calkin algebra, is d-symmetric.

Remark 3.4. $A \in L(H)$ is essentially d-symmetric if and only if $\overline{[\pi(A), \mathcal{C}(H)]}$ is self-adjoint.

Theorem 13. Let $A \in L(H)$ be essentially $d$-symmetric. Then, $A$ is $d$-symmetric if and only if $A$ is p-symmetric.

Corollary 14. An essentially $d$-symmetric weighted shift $S\left(S e_{n}=\right.$ $\left.\omega_{n} e_{n+1}\right)$ is $d$-symmetric, if and only if, it satisfies the total products condition, that is,

$$
\Sigma_{k} w_{k} \cdot w_{k+1} \ldots w_{k+n}=\infty .
$$

Example 3.2. We define our operator $A$ as follows:

Let $\left(e_{k}\right)$ be an orthonormal basis of $H$, and set

$$
A e_{k}=\frac{1}{\left(k^{2}+1\right)} e_{k+1} \text { for all } k \in \mathbb{N}(\mathbb{Z}) .
$$

$A$ is essentially normal, hence $A$ is essentially d-symmetric. But $A$ is not psymmetric.

Example 3.3. Let $\left(e_{k}\right)$ be an orthonormal basis of $H$. Set $A e_{n}=a_{n} e_{n+1}$ for $n=1,2, \cdots$, where

$$
a_{n}=\left\{\begin{array}{lll}
2, & \mathrm{n} & \text { odd } \\
\frac{1}{2}, & \mathrm{n} & \text { even }
\end{array}\right.
$$

It is easily seen that $A$ satisfies the total products condition :

$$
\Sigma_{k} w_{k} \cdot w_{k+1} \ldots w_{k+n}=\infty,
$$

for all $n \in \mathbb{N}$. Then $\{A\}^{\prime} \cap C_{1}(H)=\{0\}$, and so $A$ is p-symmetric. But $A$ is not essentially d-symmetric. 
Remark 3.5. The unilateral shift is essentially d-symmetric but is not essentially normal.

Proposition 3.2. Let $A$ and $B$ be two-essentially d-symmetric operators such that $\sigma_{e}(A) \cap \sigma_{e}(B)=\phi$. Then $A \oplus B$ is also essentially d-symmetric.

The following result is an immediate consequence of Theorem 9 and [12].

Corollary 15. Let $A \in L(H)$. Then $A$ is essentially d-symmetric in each of the following cases:

1) $A^{*} A-A A^{*}$ is compact.

2) $A^{*} A-I$ or $A A^{*}-I$ is compact.

3) $P(A)$ is cyclic subnormal operator.

4) $P(A)$ is normal and $A$ is essentially hyponormal.

\section{Conclusion}

In this, paper we are interested to the class of operators $A \in L(H)$, which satisfy the following property $A T=T A$ implies $A T^{*}=T^{*} A$ for all $T \in C_{1}(H)$ (trace class operators). Such operators are called p-symmetric. We give some basic properties concerning this class of operators. We also show that if $P(A)$ is cyclic subnormal for some polynomial $P$, then $A$ is d-symmetric. This leads us to establish the range kernel orthogonality for certain non normal derivation. We present examples and counterexamples and information about special cases. We obtain new results concerning the intersection of the kernel and the closure of the range of an inner derivation. We introduce the class of essentially dsymmetric operators. Finally, We mention the following open problems:

1) Is the set $\mathcal{F}=\{A+K: A$ p-symmetric, $K$ compact $\}$ norm closed? norm dense in $\mathrm{L}(\mathrm{H})$ ?

2) Characterizes the class of essentially d-symmetric operators?

3) Which weighted shift are essentially d-symmetric?

\section{Acknowledgments}

The authors would like to express their thanks to Professor Omar ALEHYANE, Professor Abdelkhalek FAOUZI and Professor El Hassan ZEROUALI for excel- 
lent cooperation, for encouraging our interest in this problems and for helpful suggestions.

The authors wish to thank the referee for his/her careful reading of the paper and for his useful comments.

\section{References}

[1] J.H. Anderson, On normal derivations, Proc. Amer. Math. Soc., 38 (1973), 135-140.

[2] J.H. Anderson, J.W. Bunce, J.A. Deddens and J.P. Williams, $C^{*}$-algebras and derivation ranges, Acta. Sci. Math., 40 (1978), 211-227.

[3] C. A. Berger, B.I. Shaw, Self-commutators of multicyclic hyponormal operators are always trace class, Bull. Amer. Math. Soc., 79 (1973), 1193-1199.

[4] S. Bouali and J. Charles, Extension de la notion d'opérateur d-symétrique. I, Acta. Sci. Math. (Szeged), 58(1993), 517-525.

[5] S. Bouali and J. Charles, Extension de la notion d'opérateur d-symétrique. II, Linear algebra and its applications., 225 (1995), 175-185.

[6] S. Bouali and Y. Bouhafsi, On the range-kernel orthogonality and p-symmetric operators, Math. Inequal. Appl., 9 (2006), 511-519.

[7] S. Bouali and Y. Bouhafsi, p-symmetric operators and the range of a subnormal derivation,Acta. Sci. Math(Szeged), 72 (2006), 701-708.

[8] S. Bouali and M. Ech-chad, Generalized d-symmetric operators, II, Canad. Math. Bull., 54 (2011), 21-27.

[9] P. A. Fillmore, J. G. Stampfli, and J. P. Williams, On the essential numerical range, the essential spectrum, and a problem of Halmos, Acta Sci. Math., 33 (1972), 179-192.

[10] B.C. Gupta and P.B. Ramanujan, A note on d-symmetric operators, Bull. Austral. Math. Soc., 23 (1981), 471-475.

[11] Y. Ho, Commutants and derivation ranges, Tôhoku Math. J., 27 (1975), 509-514.

[12] F. Kittaneh, On the structure of polynomially normal operators, Bull. Austral. Math. Soc., 30(1984), 11-18.

[13] S. Mecheri. Generalised p-symmetric operators, Math. Proc. R. Ir. Acad., 2 (2004), 173175 .

[14] C.R. Rosentrater, Not every d-symmetric operator is GCR,Proc. Amer. Math. Soc., 81 (1981), 443-446.

[15] C.R. Rosentrater, Compact operators and derivations induced by weighted shifts, Pacific. J. Math.,104 (1983), 465-470.

[16] J. G. Stampfli, Hyponormal operators,Pacific J. Math., 12 (1962), 1453-1458.

[17] J. G. Stamplli, On self-adjoint derivation ranges, Pacific J. Math., 82 (1979), 257-277.

[18] A. Turnsěk, Orthogonality in $C_{p}$ classes, Monatsh. Math., 132 (2001), 349-354.

[19] R.E. Weber, Derivations and the trace class operators, Proc. Amer. Math. Soc.,73 (1979), 79-81. 
[20] R.E. Weber, Analytic functions, ideals and derivation ranges, Proc. Amer. Math. Soc., 40 (1973), 492-496.

[21] J. P. Williams, Finite operators, Proc. Amer. Math. Soc., 26 (1970), 129-136.

[22] T. Yoshino, Subnormal operators with a cyclic vector, Tôhoku Math. J., 21 (1969), 47-55. 